\title{
Mediación de la lectura por parte de profesoras en sus prácticas docentes: ¿cómo son los tipos de ayuda que ofrecen a sus estudiantes?*
}

Reading mediation by teachers in their teaching practices: how are the types of support they offer to their students?

Omar Davison Toro ${ }^{* *}$, María Constanza Errázuriz Cruz ${ }^{* * *}$, Liliana Fuentes ${ }^{* * * *}$, Andrea Cocio ${ }^{* * * * *}$

\section{RESUMEN}

Las prácticas docentes de lectura en Chile son poco diversas y Palabras clave: presentan mayoritariamente escaso desafío cognitivo. Por tancomprensión to, el objetivo de la investigación fue analizar los tipos de ayuda lectora, lectura, para mediar la lectura en las prácticas docentes de profesoras de mediación lectora, La Araucanía, Chile. Respecto de la metodología, el diseño fue prácticas docentes. un estudio de casos descriptivo y cualitativo y los participantes fueron 11 docentes de siete escuelas públicas, cuyas clases fueron videograbadas, para luego analizar sus tipos de ayuda para mediar la lectura. Finalmente, se identificó que se presentan de manera simultánea los tipos de ayuda: enseñar a comprender y ayudar a comprender, pero con predominio de esta última. En

* Este proyecto fue financiado por ANID Fondecyt Regular № 1170193 titulado "Concepciones sobre la lectura y su fomento de profesores de Educación Básica en ejercicio y sus estudiantes: ¿Cómo inciden en las prácticas docentes?: Análisis de una relación significativa para el desarrollo de lectura en la escuela".

Chileno. Doctor en Ciencias Humanas mención Discurso y Cultura de la Universidad Austral de Chile. Coordinador en la Fundación Ibáñez-Atkinson. Villarrica, Chile. omardavisont@gmail.com

*** Chilena. Doctora en Análisis del Discurso Hispanoamericano de la Universidad de Valladolid. Académica de la Pontificia Universidad Católica de Chile. Villarrica, Chile. cerrazuc@uc.cl

**** Chilena. Doctora en Lingüística de la Pontificia Univesidad Católica de Valparaíso. Académica de la Universidad de Chile. Santiago, Chile. lilianafuentes@uchile.cl

*n*** Chilena. Doctora en Innovación Educativa de la Universidad de Deusto. Académica de la Universidad Católica de Temuco. Temuco, Chile. acocio@uct.cl 
este sentido, es posible constatar que los docentes se han centrado más en motivar que en desarrollar estrategias.

\begin{abstract}
The teaching practices of reading in Chile are little diverse and present mostly scarce cognitive challenge. Thus, the objective of this research was to analyse the types of support to mediate the reading in teaching practices of teachers in La Araucanía Region, Chile. Regarding the methodology, the design was a study of descriptive and qualitative cases and the participants were 11 teachers from 7 public schools which classes were videotaped to then analyse their types of support to mediate the reading. Finally, it was identified that the types of support such as teaching to understand and helping to understand are presented simultaneously being the latter the most predominant. In this sense, it is possible verify that the teachers have focused on motivating than to develop strategies.
\end{abstract} Keywords: reading mediation, reading, reading comprehension, teaching practices. 


\section{Introducción}

Uno de los propósitos de una educación de calidad es el desarrollo de las competencias comunicativas, no solo por el hecho de que sean relevantes en sí mismas, sino debido a que el aprendizaje en todos los ámbitos del conocimiento está supeditado a ellas (Bazerman et al., 2012; Carlino, 2013; Street y Street, 1995; Tolchinsky y Simó, 2001). A esto se añaden los efectos cognitivos beneficiosos de la cantidad de lectura en la mente de las personas (Cunningham y Stanovich, 1998) y que el desarrollo de la literacidad es indispensable para la formación de ciudadanos responsables en sociedades democráticas y sostenibles (Cassany, 2006; Organización de las Naciones Unidas para la Educación, la Ciencia y la Cultura [Unesco], 2017) y para la emancipación de las personas (Freire, 2012). Así lo señala también el Plan Nacional de la Lectura chileno, al declarar que lectura es un derecho social (Consejo Nacional de la Cultura y las Artes, 2015).

No obstante, a pesar de la importancia de estas competencias para la formación escolar, se presentan deficiencias tanto en estudiantes del sistema escolar chileno como de Pedagogía, lo que ha sido confirmado por varias evaluaciones como las últimas PISA y PIRLS (Agencia de Calidad de la Educación [ACE], 2017; Organisation for Economic Co-operation and Development [OECD], 2016). Del mismo modo, Errázuriz et al. (2020a) y Gallego et al. (2019) constataron que estudiantes chilenos de educación básica de las regiones de La Araucanía y Tarapacá, respectivamente, tenían un bajo rendimiento lector, el cual decrecía a medida que pasaban de curso junto con sus actitudes lectoras.

Asimismo, los estudiantes de programas de formación inicial docente también parecen presentar dificultades en este ámbito. Por ejemplo, Aguilar et al. (2016) y Errázuriz $(2017$; 2019) corroboraron que el desempeño y las concepciones acerca de la escritura de estudiantes de Pedagogía Básica de la Araucanía y Los Ríos eran limitados. En este sentido, incluso, no solo las pruebas estandarizadas y las investigaciones muestran estas falencias, sino que son los mismos docentes quienes reconocen que requieren aprender más de la enseñanza de la literacidad (Rodríguez et al., 2016).

Por último, también hay evidencias que señalan que las prácticas docentes de desarrollo de la literacidad de profesores chilenos 
de educación básica han demostrado ser poco diversas y más bien reproductivas y de bajo desafío cognitivo (Bustos et al., 2017; Iturra, 2015; Manzi et al., 2011; Medina et al., 2014;). En efecto, de acuerdo con Bustos et al. (2017), Iturra (2015) e Iturra et al. (2019) el patrón discursivo más frecuente en estas prácticas es el de InterrogaciónRespuesta-Evaluación (IRE), es decir, predominan las interacciones monológicas, las preguntas formuladas son principalmente reproductivas y los objetivos de lectura no son explicitados, o bien, son vagos, lo que no permitiría construir aprendizajes de manera dialógica y colectiva (Wells y Barberán, 2001), o en términos de Mercer (2001), interpensar juntos.

Las evidencias expuestas son preocupantes, especialmente, si consideramos que los estudiantes que muestran actitudes positivas hacia la lectura tienden a ser buenos lectores y tener un mayor rendimiento académico (Errázuriz et al., 2020b; Guthrie et al., 2007; Organización para la Cooperación y el Desarrollo Económicos [OCDE], 2014; Orellana y Baldwin, 2018; Petscher, 2010; Stutz et al., 2016; Valenzuela et al., 2015) y que contamos con diversos datos que dan cuenta del nivel de inequidad y segregación de nuestro sistema educativo (Programa de las Naciones Unidas para el Desarrollo [PNUD], 2017; Unesco, 2013; Valenzuela et al., 2014).

Por consiguiente, si bien por una parte disponemos de evidencias acerca de las prácticas docentes de lectura en la evaluación docente, estas no han sido desarrolladas en contextos ecológicos (Bustos et al., 2017; Iturra et al., 2019) y, por otra, los estudios referidos a aquellas prácticas auténticas han sido llevados a cabo en regiones del centro de país (Bustos et al., 2017; Medina et al., 2014); es por ello que el objetivo general de esta investigación ha sido analizar los tipos de ayuda para mediar la lectura en las prácticas docentes de profesores de escuelas públicas de la Región de La Araucanía, Chile. De este modo, distinguiendo las modalidades que se presentan tanto en el "ayudar a comprender" como en el "enseñar a comprender" (Sánchez et al., 2010), se podrán ofrecer insumos para comprender la situación actual de las prácticas docentes en Chile y, así, aportar a mejorar la calidad de estas y de la formación docente. 


\section{Marco teórico}

\section{La mediación de la lectura en las disciplinas}

Sabemos que la docencia de excelencia puede tener efectos positivos sobre el aprendizaje de los estudiantes, la solidez de su formación y la inclusión de aquellos más desfavorecidos (Barber y Mourshed, 2008; Liston et al., 2008; Manzi y García, 2016). En este sentido, como la comprensión de las lecturas que llevan a cabo los estudiantes se negocia y construye en comunidad es el docente quien, como lector experto, debe traspasar progresivamente su experiencia y el monitoreo al lector inexperto (Bustos et al., 2017) y, por ello, media los textos y configura un espacio de referencias en común (Chartier y Hébrard, 2000). No obstante, según Rockwell (2001), hay prácticas de enseñanza de la lectura que alejan a los estudiantes de la cultura escrita y que los excluye, al no mediar los textos y relacionarlos con su experiencia y no orientar un sentido.

Según algunos estudios, esto sucede porque se ha observado que los estudiantes del sistema escolar y de Pedagogía presentan propósitos de lectura principalmente utilitarios, es decir, leen solo para la evaluación, pues no leen habitualmente ni efectúan una construcción personal de los textos (Muñoz et al., 2016; Yubero et al., 2004). Asimismo, como un lector motivado dedica más tiempo y esfuerzo a la lectura (Guthrie et al., 2007), se ha constatado que el compromiso lector de los estudiantes es determinante en sus resultados de comprensión lectora, incluso aún más que las estrategias de lectura (Errázuriz et al., 2020b; Orellana y Baldwin, 2018; Petscher, 2010; Valenzuela et al., 2015). En ese sentido, los perfiles lectores de los estudiantes también están vinculados con su rendimiento (Errázuriz et al., 2020a; Mateos et al., 2017; Schraw y Bruning, 1999), pues aquellos que manifiestan concepciones de lectura más epistémicas obtienen más altos resultados en lectura, muestran más compromiso por la tarea y una visión más crítica del texto que aquellos apegados a concepciones reproductivas. Lo anterior es explicado por el modelo de Rosenblatt (1985), quien ha definido la lectura como un proceso de transacción entre el lector y el texto, de acuerdo con los objetivos que la orienten, lo que permite construir un significado. Por ende, el lector más activo puede construir una comprensión más profunda según su propósito. 
De acuerdo con lo anteriormente señalado, un lector motivado es gravitante para el desempeño lector. En este sentido, se ha identificado el efecto que pueden tener las concepciones y hábitos de lectura del profesorado sobre sus prácticas de enseñanza y mediación lectora en el aula, y sobre el modelamiento de las actitudes y los hábitos lectores del alumnado (Errázuriz et al., 2019; Foorman y Schatschneider, 2003; Morrison et al., 1999; Munita, 2016; Orellana y Baldwin, 2018; Schraw y Bruning, 1999; Sulentic-Dowell et al., 2006). En efecto, se ha constatado que el profesorado escolar chileno que presenta perfiles lectores más epistémicos asocia las prácticas de lectura con el placer, media la lectura de sus estudiantes, se interesa por motivarla y son más inclusivos (Errázuriz et al., 2020a; Errázuriz et al. 2019). No obstante, sabemos que aún existe una resistencia por parte de los profesores de otras disciplinas respecto de enseñar la lectura, pues creen que es una habilidad propia del ámbito de lengua (Carlino, 2005; Muñoz et al., 2016) $\mathrm{y}$ no necesariamente una actividad frecuente en todas las disciplinas; por tanto, se impone un enfoque generalista y no contextualizado de la lectura (Shanahan y Shanahan, 2012).

Desde otra perspectiva, se ha señalado que los jóvenes sí leen y sostienen sus propias prácticas de lectura, sin embargo, en general no son consideradas en la sala de clases (Winocur, 2015). Por tanto, y siguiendo a Gaete-Moscoso (2019) y a Zavala (2011), es necesario también validar las prácticas de lectura híbridas de los estudiantes y docentes.

En este sentido, a partir de la propuesta de Sánchez et al. (2010), hemos considerado dos tipos de mediaciones de lectura que nos parecen relevantes de analizar. Por un lado, enseñar a comprender (ayudas frías) se relaciona con enseñar explícitamente al alumnado las estrategias de lectura disciplinar para decodificar y comprender el contenido del texto, es decir, las competencias retóricas para manejar los discursos y los diversos géneros discursivos. Por otro lado, ayudar a comprender (ayudas cálidas) es un modo de enseñanza más implícito y está dirigido a acompañar y motivar a los estudiantes en la lectura de cierto texto, creando un contexto de lectura facilitador al socializar el propósito de lectura y experiencias personales de lectura. Por ejemplo, un maestro que usa ayudas frías guía la lectura de los estudiantes, mostrándoles explícitamente estrategias de lectura para comprender el texto, relacionar la información y reflexionar metacognitivamente, 
por lo tanto, sus intereses docentes residen en los procesos cognitivos del alumnado. En cambio, un docente que despliega ayudas cálidas se enfoca en que el ambiente de lectura sea agradable y alienta a sus alumnos para motivarlos, por lo que sus intereses docentes están relacionados con procesos emocionales y una visión más sociocultural de la lectura en que intervienen los contextos del lector, el autor y el texto (Cassany, 2006), así, al compartir marcos de interpretación y objetivos comunes, pueden conformar comunidades de lectores, de aprendizaje y de práctica (Wenger, 1998). Sin embargo, ambas mediaciones son necesarias para desarrollar habilidades y actitudes lectoras y realmente son complementarias y compatibles, pues se puede ayudar a comprender para enseñar a comprender.

\section{Método}

La presente investigación se circunscribe como un estudio de casos múltiples (Flyvbjerg, 2006), descriptivo de corte cualitativo e interpretativo (Merriam y Tisdell, 2009).

\section{Participantes}

Las participantes corresponden a 11 profesoras de Educación Básica de siete escuelas públicas de la Región de La Araucanía de Chile de tres ciudades (Temuco, Villarrica y Pucón), quienes fueron elegidas aleatoriamente y por disponibilidad desde una muestra representativa de 338 docentes de 18 establecimientos educativos de las cuatro comunas más pobladas del territorio: Temuco, Villarrica, Angol y Pucón (Ministerio de Educación de Chile [Mineduc], 2016). Cabe señalar que esta región tiene los mayores índices de pobreza del país (Ministerio de Desarrollo Social, 2016) y una realidad multicultural que no ha sido suficientemente abordada desde un enfoque intercultural (Alarcón et al., 2018).

Al momento del estudio, las participantes tenían un promedio de 20 años de experiencia en el aula y siete de ellas contaba con estudios de posgrado. Asimismo, 10 de ellas son profesoras de Educación Básica y una es profesora de Ciencias Sociales; 3 son miembros de un pueblo originario, específicamente, mapuche; y la totalidad realiza clases en distintas disciplinas de $3^{\circ}$ a $8^{\circ}$ año básico. Algunos de los datos se especifican en la Tabla 1. 


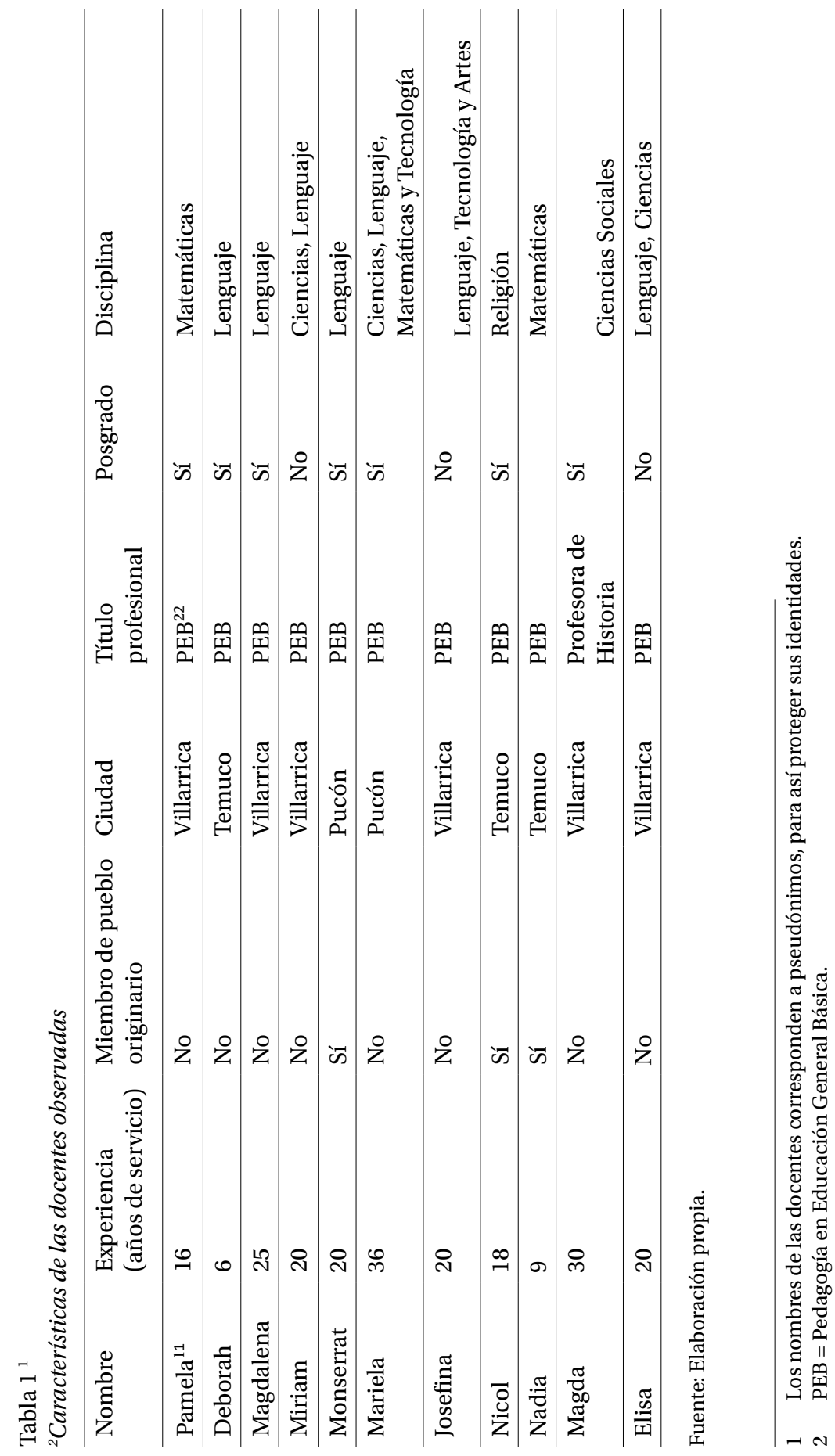




\section{Procedimientos}

A partir de lo anteriormente descrito, videograbamos las prácticas docentes de las participantes ya mencionadas que presentaran actividades de lectura en distintas disciplinas. En particular, correspondieron a tres sesiones de clases de 45 minutos de cada docente, es decir, 33 clases en total, lo que equivale 1.485 minutos o 24,75 horas de clases lectivas. Además, registramos notas de campo complementarias y transcribimos el audio de los videos.

Respecto del procedimiento de análisis, consideramos en parte la propuesta de Sánchez et al. (2010), desde un enfoque cualitativo. Así, en primer lugar, segmentamos las interacciones en ciclos, la unidad mínima compuesta por una serie de interacciones en torno al acuerdo entre dos o más individuos. En segundo lugar, identificamos la categoría de tipos de ayuda y sus dimensiones: ayudar a comprender y enseñar a comprender y en qué modalidades se manifiestan. Estas unidades y dimensiones fueron identificadas en el corpus por medio del programa Dedoose, calibradas por el equipo investigador y luego analizadas e interpretadas cualitativamente. En este sentido, se efectuó una codificación inicial para acordar y validar los primeros códigos. Posteriormente, en un segundo análisis por parte de los miembros del equipo, se llevó a cabo una codificación central para revisar y calibrar la codificación y establecer los códigos definitivos, con el fin de pasar a la fase de interpretación de los datos y codificación final. Además, durante los diferentes procesos de codificación se escribieron memos analíticos que fueron considerados en la interpretación de los datos. En consecuencia, los códigos se sometieron al menos a tres calibraciones por parte de diferentes miembros del equipo.

\section{Resultados}

De acuerdo con el análisis de las prácticas pedagógicas de lectura que llevó a cabo el grupo de profesoras estudiado, un primer resultado muestra que se desplegaron ayudas vinculadas tanto al "ayudar a comprender" como al "enseñar a comprender". En específico, pese a la coexistencia de ambos tipos de ayuda, constatamos que en el caso del "ayudar a comprender" se presentaron 172 ciclos, mientras que en el de "enseñar a comprender" el resultado fue de 122 ciclos. De este 
modo, se presentaría una mayor tendencia a emplear las ayudas enfocadas en el "ayudar a comprender".

En efecto, aun cuando la propia literatura advierte acerca de la importancia de favorecer ambos tipos de ayuda para una mejor comprensión, la tendencia identificada estaría mostrando, de modo incipiente, que estas profesoras efectivamente se preocupan por configurar un ambiente más propicio para las prácticas de lectura. Siguiendo los términos que proponen Sánchez et al. (2010), estaríamos en presencia de profesoras que en sus pautas de enseñanza visibilizan ayudas más "cálidas" por sobre acudir a la enseñanza directa de algunas estrategias de comprensión lectora, que son las ayudas "frías", como plantean estos investigadores.

En el caso específico del "ayudar a comprender", identificamos tres modalidades. En primer lugar, algunas profesoras se encargan de transmitir un mensaje alusivo a lo importante que es leer y los beneficios que supone esta práctica:

Vamos a comenzar entonces nuestra clase de hoy, donde vamos a aprender nuevamente en base a la lectura. Ustedes saben que la lectura es la clave del éxito en el futuro, ¿̇o no? (Magdalena, Lenguaje, $5^{\circ}$ y $6^{\circ}$ año básico).

En segundo lugar, constatamos el interés por generar un ambiente adecuado para desarrollar la lectura en el marco de un respeto mutuo, tal como lo ilustra este fragmento:

Primero hacemos el ejercicio de quedarnos callados, así la persona que participe sabe que va a ser escuchada. ¿Quién va a comenzar? ¿Pedro, quieres comenzar? (Miriam, Ciencias, Lenguaje, $5^{\circ}, 6^{\circ}, 7^{\circ} \mathrm{y}$ $8^{\circ}$ año básico).

La última modalidad, alude a factores de índole más motivacional y emocional, es decir, advertimos la presencia reiterada - en el transcurso de toda la clase- de ciclos donde la profesora refuerza la motivación y compromiso del alumnado con la actividad. Por una parte, mientras algunas profesoras felicitan a sus alumnos:

Muy bien, le damos un aplauso a Benjamín por su lectura (Magdalena, Lenguaje, $5^{\circ}$ y $6^{\circ}$ año básico).

otras lo hacen destacando las respuestas recibidas: 
Muy bien, amigo, muy buena respuesta. Reforestar, significa a que vamos a cortar esos árboles, porque ya están grandes, o ya están viejos, pero vamos a poner árboles (Elisa, Lenguaje, Ciencias, $3^{\circ}$ año básico).

Como sostienen algunos autores (Munita, 2016; Orellana y Baldwin, 2018; Sánchez et al., 2010), el empleo de refuerzos positivos es importante dado que esto contribuye a favorecer en los estudiantes una actitud positiva frente a la lectura y un alto autoconcepto como lectores. En efecto, desde un enfoque asociado a la mediación lectora (Chambers, 2007; Petit, 2003), también se ha destacado la importancia de que el profesorado indague si los textos que proponen son del gusto de los alumnos, como lo ilustra el caso de Monserrat:

Profesora: ¿Les gustó el cuento?

Estudiantes: (Todos al unísono) ¡Sí!

Profesora: Qué bueno, porque hay otro más lindo, porque miren como está el libro. Es porque muchos lo han leído. ¿Ya? Se llama Cuentos chilenos para niños. ¿Les gustó? (Monserrat, Lenguaje, $5^{\circ}$ año básico).

Como explicamos anteriormente, estos resultados sugieren que el "ayudar a comprender" no se agota en una modalidad particular; más bien, constatamos que coexisten diversas alternativas. Sin embargo, en una lectura más global, podríamos argumentar que gracias a estas ayudas, las profesoras estudiadas perciben al estudiante como un sujeto lector que no solo debe adquirir y desarrollar un conjunto de habilidades cognitivas para lograr una comprensión adecuada, sino que también requiere reforzar continuamente su autoestima lectora, dando oportunidades para dialogar y plantear su opinión.

En una perspectiva complementaria, si bien el "ayudar a comprender” se caracteriza por el propósito de crear un ambiente favorable para la lectura, es necesario destacar que, al momento de desarrollar una sesión de lectura, un número importante de las profesoras analizadas sí se preocupa por motivar la actividad. No obstante, no necesariamente lo hacen a través de contar su experiencia de lectura previa con el texto a trabajar. De este modo, de acuerdo con la conceptualización que alude a la dimensión del docente como sujeto lector (Munita, 2017), se podría argumentar que esta es una dimensión que no apa- 
rece de forma nítida en las prácticas analizadas, pese a que también es una fórmula que contribuye a favorecer el "ayudar a comprender", tal como ha sido constatado por ciertos autores (Errázuriz et al., 2019, Munita, 2016; 2017).

En el caso de las ayudas vinculadas con el "enseñar a comprender", cuyo propósito es favorecer la comprensión del texto, tal como se planteó anteriormente con el "ayudar a comprender", también se presenta la coexistencia de diversas modalidades, como explicaremos a continuación.

En primer término, desde una perspectiva general, observamos que el empleo de la pregunta es un recurso bastante utilizado por las profesoras. Ahora bien, cabe precisar que estas preguntas aparecen en distintos momentos de la clase y, por ende, su formulación responde a diversos propósitos. Sin embargo, es necesario precisar que gran parte de ellas se mueve entre dos polos: intentan obtener información de un aspecto muy general y causal del texto, o bien, se intenta pesquisar información muy puntual, dado que se enuncian preguntas del tipo “¿y porqué sucedió esto?”, o también “¿por qué el texto dice esta palabra?". Bajo nuestro punto de vista, subrayar esta dimensión radica en señalar que el escaso número de preguntas identificadas sobre aspectos propiamente internos del texto o relevantes para la trama podría ser un indicio de que no en todas las profesoras se presenta una clara "conciencia del texto". En efecto, este factor da cuenta precisamente de "una reflexión sobre los textos que nos lleva a identificar las ideas que contiene y las relaciones que cabe establecer entre ellas" (Sánchez et al., 2010, p. 323). Por lo tanto, podríamos afirmar que probablemente solo en algunas de las prácticas observadas, las profesoras leyeron profundamente el texto "como un instrumento educativo que contiene un cierto número de posibles proyectos que pueden ser desvelados" (Sánchez et al., 2010, p. 324).

Por otro lado, en otras modalidades presentes, observamos que el trabajo de comprensión e interpretación que este grupo de profesoras intenta favorecer en sus prácticas, supone una exploración del texto desde diversos encuadres. Así, constatamos el interés de algunas profesoras por estimular la formulación de predicciones, como sucede con Elisa: 
al final sí hubo amistad, ya. Eh... ¿Qué otra de las predicciones que ustedes hicieron se cumplieron?, ¿qué otras de las que dijo alguien aquí?" (Elisa, Lenguaje y Ciencias, $3^{\circ}$ año básico).

Asimismo, la estrategia de interrogar el texto con la colaboración del alumnado también se visibiliza como otra modalidad del "enseñar a comprender":

Quiero preguntar otra cosa. Quiero saber si alguno de ustedes tiene alguna pregunta que hacer. Y síla tiene, esa pregunta tiene respuesta con nuestro cuento. ¿Cuál es su pregunta?, después la Lorena, ¿ya? (Monserrat, Lenguaje, $5^{\circ}$ año básico).

En la misma perspectiva, el propósito de distinguir con claridad si la información está explícita o bien implícita en el texto, es otra de las modalidades que algunas profesoras ponen en práctica, como lo ilustra el siguiente fragmento:

Profesora: Ya, chicos a mí me gustaría que nos quedara claro, entonces, este texto que estamos leyendo. Aparte de lo que sabemos de esa obra, nos quedaba claro que vamos a ir a buscar información que está más allá del texto, lo que nosotros hablamos en alguna oportunidad que se llama implícito, ¿ya? Nosotros sabemos que en el texto hay información que está ahí mismo. Por ejemplo, si yo le pregunto a ustedes: ¿qué hacía el león al inicio de nuestra historia? (Elisa, Lenguaje y Ciencias, $3^{\circ}$ año básico).

Esclarecer algún término o frase del texto que los estudiantes no necesariamente comprenden, también es una de las ayudas que estas profesoras ponen en práctica. En ese contexto, algunas dialogan con sus alumnos para llegar a una respuesta:

Profesora: En la número dos... Aline, ¿cuál sería el verbo?, la orden que me están dando.

Estudiante: Traza aquí.

Profesora: Traza. Ahora mi pregunta es ¿quién sabe lo que significa traza?

Estudiante: Ah, ah.

Estudiante 1: Correr.

Estudiante 2: Traza es... hacer una línea.

Profesora: Ya. Bien (Elisa, Lenguaje y Ciencias, $3^{\circ}$ año básico). 
En otras prácticas, reconocemos el propósito de señalar el significado en cuestión, pero aprovechando la instancia para socializar la utilidad de la lectura como un recurso fundamental para la adquisición de vocabulario:

¿Se dan cuenta?, a través de la lectura y la comprensión de la lectura nosotros podemos encontrar el significado de algunas palabras que no conocemos. No necesitamos siempre el diccionario, a veces con entender basta (Elisa, Lenguaje y Ciencias, $3^{\circ}$ año básico).

Por otro lado, si la aclaración de alguna palabra o frase es un indicio de cómo se trabaja una parte específica del texto, también se presenta el interés por manejarlo desde una perspectiva más global y, en ese plano, se ubican las ayudas orientadas a explicar alguna función o característica distintiva del texto que está siendo trabajado. Por ejemplo, para explicar la particularidad que reviste un libro álbum, Miriam destaca la presencia de las imágenes como una característica que se debería tener presente:

Profesora: Que tienen muchas imágenes, ¿cierto? Ahora vamos a ver los libros álbum.

Estudiante: ¿Qué son?

Profesora: Son los que más dicen las imágenes. Tienen muy pocas letras.

Estudiante: $\mathrm{mmm} .$.

Profesora: Pero dicen mucho las imágenes. Para eso hay que poner mucha atención en los detalles, para conocer bien un libro álbum. Lo que van a hacer ustedes es lectura de un libro álbum, pero en grupo, así que les invito a ponerse de pie. Dos grupos de tres. Y un grupo de cuatro personas (Miriam, Lenguaje, $5^{\circ}$ año básico).

Como indican Sánchez et al. (2010), durante la fase de lectura, las ayudas enfocadas en la enseñanza de estrategias son un componente relevante para el propósito de favorecer una adecuada comprensión; no obstante, este principio se enriquece sustantivamente en la fase de poslectura, dado que es el momento para consolidar lo aprendido. En esa línea, también se presentan varias alternativas. Por una parte, mientras algunas profesoras optaron por que los alumnos escribieran resúmenes, esquemas, subrayaran ideas e incluso dibujaran, para des- 
pués compartirlos con los demás compañeros; otras prefirieron dialogar con sus alumnos con el propósito de favorecer reflexiones de tipo metacognitivo, como en el caso de Deborah:

Profesora: A ver... Vicente, cuando tú leíste un texto, para poder comprenderlo, ¿qué fue lo que hiciste?

Estudiante: volver a leer (Deborah, Lenguaje, $5^{\circ}, 6^{\circ}$ y $7^{\circ}$ año básico).

Por último, en la línea de estimular el diálogo con los alumnos una vez finalizada la lectura, también observamos cómo algunas profesoras abren un espacio para que el alumnado explique lo que aprendió, o bien, manifieste alguna opinión más personal. Como se ilustra en los siguientes fragmentos:

Profesora: Ya chiquillos vamos... con esto terminaría la clase de hoy. Solo quiero dos opiniones.

Profesora: ¿Qué les pareció?, o ¿que aprendieron en esta clase? A ver... hay gente que no ha participado hoy día. Quiero escucharlos... a ver, Seba, Sebita.

Estudiante: ¿Ah?

Profesora: ¿Qué te pareció?, ¿qué aprendiste?, ¿qué fue lo nuevo?, ¿qué te vas a llevar a casa hoy día? A ver... (Magdalena, Lenguaje, $5^{\circ}$, $6^{\circ}$ año básico).

Profesora: ¿Comprendieron la historia del pato y la muerte?

Estudiante: Sí.

Profesora: ¿Quién tiene una opinión de esa historia?, ¿les gustó?, o les parece... a ver Gladys.

Estudiante: A mí no me gustó, que hubiera muerto la muerte, en vez del pato (Miriam, Ciencias y Lenguaje, $5^{\circ}, 6^{\circ}, 7^{\circ}$ y $8^{\circ}$ año básico).

En consecuencia, en la perspectiva del "enseñar a comprender", los resultados evidenciarían que este grupo de profesoras pone en práctica diversas modalidades asociadas con el objetivo de este tipo de ayuda. Si bien lo hacen con diversos énfasis, de igual modo cabe precisar que habría un factor común que se presenta en la mayoría de las prácticas analizadas. Esto es que, al momento de emplear una de las modalidades del enseñar a comprender, las docentes no necesariamente desti- 
nan un momento para ejercer un modelaje previo de la estrategia. Por lo tanto, las oportunidades que tendría el estudiantado durante la clase para observar cómo la profesora utiliza determinada estrategia no se presentan con la frecuencia que sería deseable, dada la importancia que posee el docente como lector experto en su traspaso progresivo de su experiencia y monitoreo al lector inexperto (Bustos et al., 2017).

Finalmente, las observaciones y análisis presentados nos llevan a plantear que el tratamiento de la lectura por parte del grupo de profesoras estudiado, supone el despliegue de un conjunto de repertorios pedagógicos donde se alternan distintos modos tanto para "enseñar a comprender" como para "ayudar a comprender", pero con predominio de este último. No obstante, cabe profundizar en otras aristas derivadas de este planteamiento inicial.

\section{Discusión y conclusiones}

Asumiendo un punto de vista más general, en primer lugar, señalamos que el conjunto de resultados obtenidos permitiría concluir que las profesoras estudiadas despliegan en sus prácticas momentos para "ayudar a comprender" así como para "enseñar a comprender". En efecto, operacionalizar la categoría de los tipos de ayuda nos permitió relevar una serie de modalidades y, con ello, constatar que efectivamente las profesoras no acuden a una sola estrategia, sino que evalúan estratégicamente qué tipo de ayuda proporcionar. Como apunta Sánchez et al. (2010), "no es posible dar todas las ayudas, lo que supone que, para ayudar, hay que elegir” (p. 65). De ahí la frecuencia y énfasis con que se presentan determinadas modalidades en el transcurso de la clase.

Por otro lado, que el número de ciclos vinculado con el "ayudar a comprender" fue mayor que el presentado por el "enseñar a comprender, es un resultado que nos lleva a plantear dos observaciones. En primer lugar, para las docentes participantes es relevante la configuración de un ambiente propicio y grato para leer y, por ello, proporcionan ayudas orientadas a relevar los beneficios de la lectura, así como motivar el compromiso del alumno con la actividad, entre otras modalidades. En esa línea, dado que en algunas prácticas se releva el interés por favorecer la participación del alumnado no solo a través de las preguntas, sino también poniendo en escena sus propios gustos e intereses, 
nuestra segunda observación es que dichas prácticas estarían transitando hacia un repertorio más vinculado con la figura del mediador de la lectura. De hecho, en otro estudio hemos constatado que algunas de estas profesoras efectivamente se identifican con este rol (Errázuriz et al., 2019). Como sugieren otras investigaciones (Chambers, 2007; Munita, 2016; 2017) y se ratifica en un conjunto de documentos curriculares y políticas públicas de promoción lectora (Consejo Nacional de la Cultura y las Artes, 2015), la noción del mediador de la lectura está cobrando cada vez más relevancia, probablemente, por la necesidad de elevar el gusto y desempeño lector en nuestro país.

No obstante lo anterior, cabría notar un interesante contrapunto, dado que en general las profesoras proporcionaron escasas ayudas que favorecieran una comprensión crítica y metacognitiva del texto. Por ejemplo, se observaron escasas referencias en cuanto a la intención y el esquema retórico de los textos. Probablemente, esto se pueda deber a que a las docentes no siempre les fue posible profundizar previamente en el texto, para así identificar las ideas y relaciones que este contiene en términos disciplinares o, como lo plantean Sánchez et al. (2010), incrementar su "conciencia del texto". Sin embargo, es necesario también considerar los contextos de estas profesoras, dada la complejidad de tomar decisiones estratégicas por parte del profesorado al momento de tratar un texto, pues sus escuelas son públicas y la mayoría de sus estudiantes, desaventajados, por lo que eso podría explicar en parte sus opciones sobre motivar a los estudiantes con la lectura como propósito primordial.

Por ello, dada la relevancia que pueden tener los perfiles lectores del profesorado sobre sus prácticas de enseñanza y de mediación lectora en el aula (Errázuriz et al., 2019; Orellana y Baldwin, 2018; Sulentic-Dowell et al., 2006); creemos pertinente advertir que el factor "conciencia del texto" debería ser un objeto de estudio más persistente en el ámbito de las prácticas de lectura y en la formación docente, debido al rol que juega en la eficacia de los tipos de ayuda que decide proporcionar el docente.

Asimismo, cabe destacar que hemos podido comprobar cómo los tipos de ayuda y sus modalidades identificadas en las docentes observadas no se relacionan con ninguna área del currículo en particular, lo que resulta desconcertante. Sin embargo, como las profesoras parti- 
cipantes son docentes de Educación General Básica y algunas dictan varias asignaturas, el poder desarrollar ciertas habilidades de lectura específicas que suponen las disciplinas, como proponen Shanahan y Shanahan (2012), resulta al menos complejo.

Finalmente, en términos de la proyección de este tipo de estudios, creemos necesario seguir profundizando en el análisis y comprensión de los tipos de ayudas en las prácticas docentes, sin perder de vista las modalidades que se ponen en juego, puesto que no solo contribuyen al propósito de elevar el desempeño lector de los estudiantes, sino que también son insumos relevantes para efectos de avanzar en la indagación de otras aristas como, por ejemplo, el modo en que el profesorado considera los fondos de conocimientos de su alumnado (Llopart y Esteban-Guitart, 2018), negocia con ellos las prácticas de lectura y coconstruye comunitariamente (Wenger, 1998) los sentidos de los textos. De esta manera, será posible visibilizar las expectativas implícitas acerca de la literacidad de los docentes (Zavala, 2011), transparentando así las prácticas de lectura y su enseñanza. A nuestro modo de ver, esa base permitiría seguir favoreciendo la figura del profesor y sus vínculos con la mediación lectora, en el horizonte de articular un proceso de formación más inclusivo y de mayor calidad.

\section{Referencias bibliográficas}

Agencia de Calidad de la Educación. (2017). Estudio Internacional de Lectura PIRLS 2016: Presentación Nacional de Resultados. ACE. http://www.agenciaeducacion.cl/noticias/1-4-estudiantes-chile-logran-los-mejores-resultados-estudio-internacional-lectura/

Aguilar, P., Albarrán, P., Errázuriz, M. C., y Lagos, C. (2016). Teorías implícitas sobre la escritura: Relación de las concepciones de estudiantes de Pedagogía Básica con la calidad de sus textos. Estudios Pedagógicos (Valdivia), 42(3), 7-26. http://dx.doi. org/10.4067/S0718-07052016000400001

Alarcón, A., Castro G., M., Astudillo, P., y Nahuelcheo, Y. (2018). La paradoja entre cultura y realidad: el esfuerzo de criar niños y niñas mapuche en comunidades indígenas de Chile. Chungará, 50(4), 651-662. http://dx.doi.org/10.4067/S071773562018005001601 
Barber, M. y Mourshed, M. (2008). Cómo hicieron los sistemas educativos con mejor desempeño del mundo para alcanzar sus objetivos. CINDE McKinsey y Company. http://educacion2020. cl/wp-content/uploads/2012/10/como_hicieron_los_sistemas_educativos_con_mejor_desempeno_del_mundo_ para_alcanzar_sus_objetivos.pdf

Bazerman, C., Dean, C., Early, J., Lunsford, K., Null, S., Rogers, P., y Stansell, A. (Eds.). (2012). International advances in writing research: Cultures, places, measures, Perspectives on writing. The WAC Clearinghouse and Parlor Press.

Bustos, A., Montenegro, C., Tapia, A., y Calfual, K. (2017). Leer para aprender: Cómo interactúan los profesores con sus alumnos en la Educación Primaria. Ocnos. Revista de Estudios sobre la Lectura, 16(1), 89-106. http://dx.doi.org/10.18239/ocnos_2017.16.1.1208

Carlino, P. (2013). Alfabetización académica diez años después. Revista Mexicana de Investigación Educativa, 18(57), 355-381. https://www.redalyc.org/pdf/140/14025774003.pdf

Carlino, P. (2005). Escribir, leer y aprender en la universidad. Una introducción a la alfabetización académica. Fondo de Cultura Económica.

Cassany, D. (2006). Tras las líneas: Sobre la lectura contemporánea. Graó.

Chambers, A. (2007). El ambiente de la lectura. Fondo de Cultura Económica.

Chartier, A. y Hébrard, J. (2000). Saber leer y escribir: unas 'herramientas mentales' que tienen su historia. Infancia y Aprendizaje, 23(89), 11-24. https://doi. org/10.1174/021037000760088053

Consejo Nacional de la Cultura y las Artes. (2015). Política Nacional de la Lectura y el Libro 2015-2020. https://www.cultura. gob.cl/wp-content/uploads/2014/08/politica-libro-lectura-2015-2020.pdf

Cunningham, A. E. y Stanovich, K. (1998). What reading does for the mind. Journal of Direct Instruction, 1(22),137-149. https:// mccleskeyms.typepad.com/files/what-reading-does-forthe-mind.pdf

Errázuriz, M. C. (2019). Desempeño escrito de estudiantes de programas de formación inicial docente: ¿Cómo es la calidad del proceso de escritura de sus ensayos?. Len- 
gua y Habla, (23), 224-242. https://www.redalyc.org/ journal/5119/511966657012/511966657012.pdf

Errázuriz, M. C. (2017). Teorías implícitas sobre escritura académica de estudiantes de programas de formación inicial docente: ¿Inciden en el desempeño escrito? Revista Signo y Pensamiento, 36(71), 34-50. https://doi.org/10.11144/javeriana.m13.tiee

Errázuriz, M. C., Becerra, R. Aguilar, P. Cocio, A., Davison, O., y Fuentes, L. (2019). Perfiles lectores de profesores de escuelas públicas de la Araucanía, Chile: una construcción de sus concepciones sobre la lectura. Perfiles Educativos, 41(164), 28-46. https://doi.org/10.22201/iisue.24486167e.2019.164.58856

Errázuriz, M. C., Fuentes, L., Cocio, A., Davison, O., Becerra, R., y Aguilar, P. (2020a). ¿Comprendemos más los textos si nos gusta leer?: Actitudes y desempeños lectores del estudiantado de escuelas públicas de La Araucanía, Chile. Revista Brasileira de Lingüistica Aplicada, 20(3), 549-589. http://dx.doi. org/10.1590/1984-6398202015350

Errázuriz, M. C., Fuentes, L., Davison, O., Cocio, A., Becerra, R., y Aguilar, P. (2020b). Concepciones sobre la lectura del profesorado de escuelas públicas de La Araucanía: ¿Cómo son sus perfiles lectores? Revista Signos, 53(103), 419-448. http://dx.doi. org/10.4067/S0718-09342020000200419

Flyvbjerg, B. (2006). Five misunderstandings about case-study research. Qualitative inquiry, 12(2), 219-245. https://doi. org/10.1177/1077800405284363

Foorman, B. R. y Schatschneider, C. (2003). Measuring teaching practice during reading/ language arts instruction and its relation to student achievement. En S. Caughn y K. L. Briggs (Eds.), Reading in the classroom: Systems for observation of teaching and learning (pp. 1-30). Brookes Publishing.

Freire, P. (2012). Pedagogía del oprimido. Siglo XXI.

Gaete-Moscoso, R. C. (2019). Escenas de lectura: ¿qué repertorio usan los docentes que enseñan a leer para hablar de su práctica lectora? Literatura y lingüistica, (40), 209-227. http://dx.doi. org/10.29344/0717621x.40.2067

Gallego, J. L., Figueroa, S., y Rodríguez, A. (2019). La comprensión lectora de escolares de educación básica. Literatura y lingüística, (40), 187-208. http://dx.doi.org/10.29344/0717621x.40.2066 
Guthrie, J. T., Hoa, A. L. W., Wigfield, A., Tonks, S. M., Humenick, N. M., y Littles, E. (2007). Reading motivation and reading comprehension growth in the later elementary years. Contemporary Educational Psychology, 32(3), 282-313. https://doi. org/10.1016/j.cedpsych.2006.05.004

Iturra, C. (2015). La organización de la enseñanza de la lectura en las aulas chilenas. Diferencias entre profesores calificados como básicos o como competentes. Revista Javeriana, 14(2), 535548. http://dx.doi.org/10.11144/Javeriana. upsy14-2.oela

Iturra, C., Donoso, E., y Fuentes, I. (2019). La práctica pedagógica en la fase antes de la lectura en clases de comprensión de textos en enseñanza media. Literatura y lingüística, (40), 228-249. http://dx.doi.org/10.29344/0717621x.40.2077

Liston, D., Borko, H., y Withcomb, J. (2008). The teacher educator's role in enhancing teacher quality. Journal of Teacher Education, 59(2), 111-116. https://doi.org/10.1177/0022487108315581

Llopart, M. y Esteban-Guitart, M. (2018). Funds of knowledge in 21st century societies: Inclusive educational practices for underrepresented students. A literature review. Journal of Curriculum Studies, 50(2), 145-161. http://dx.doi.org/10.1080/0022 0272.2016.1247913

Manzi, J. y García, M. R. (Eds.) (2016). Abriendo las puertas del aula. Transformación de las prácticas docentes. Ediciones UC.

Manzi, J., González, R., y Sun, Y. (2011). La evaluación docente en Chile. MIDE UC.

Mateos, M., Solé, I., Martín, E., Castells, N., Cuevas, I., y GonzálezLamas, J. (2017). Perfiles de creencias epistemológicas sobre la lectura y su papel en la comprensión de textos múltiples. Electronic Journal of Research in Education Psychology, 14(39), 226-252. https://doi.org/10.25115/ejrep.39.15058

Medina, L., Valdivia, A., y San Martín, E. (2014). Prácticas pedagógicas para la enseñanza de la lectura inicial: un estudio en el contexto de la evaluación docente chilena. Psykhe (Santiago), 23(2), 1-13. https://doi.org/10.7764/psykhe.23.2.734

Mercer, N. (2001). Palabras y mentes: cómo usamos el lenguaje para pensar juntos. Planeta (GBS).

Merriam, S. B. y Tisdell, E. T. (2009). Qualitative research: A guide to design and implementation. Jossey-Bass. 
Ministerio de Educación de Chile, Mineduc. (2016). Estadísticas de la educación 2015. Centro de Estudios Mineduc. https:// centroestudios.mineduc.cl/wp-content/uploads/sites/100/2017/08/Anuario_2015.pdf

Ministerio de Desarrollo Social. (2016). Casen 2015. Ampliando la mirada sobre la pobreza y la desigualdad. http://observatorio.ministeriodesarrollosocial.gob.cl/storage/docs/casen/2015/CASEN_2015_Ampliando_la_mirada_sobre_la_ pobreza_desigualdad.pdf

Morrison, T. G., Jacobs, J., y Swinyard, W. R. (1999). Do teachers who read personally use recommended practices in their classroom? Reading Research and Instruction, 38(2), 81-100. https://doi.org/10.1080/19388079909558280

Munita, F. (2017). El sujeto lector didáctico: "lectores que enseñan y profesores que leen". Álabe. Revista de Investigación sobre Lectura y Escritura, 9(17), 1-19. https://doi.org/10.15645/ alabe2018.17.2

Munita, F. (2016). Prácticas didácticas, creencias y hábitos lectores del profesor en una escuela exitosa en la promoción lectora. Ocnos. Revista de Estudios sobre la Lectura, 15(2), 77-97. https://doi.org/10.18239/ocnos_2016.15.2.1140

Muñoz, C., Valenzuela, J., Avendaño, C., y Núñez, C. (2016). Mejora en la motivación por la lectura académica: la mirada de estudiantes motivados. Ocnos. Revista de Estudios sobre Lectura, 15(1), 52-68. https://doi.org/10.18239/ocnos_2016.15.1.941

Orellana, P. y Baldwin, P. (Eds.) (2018). Reading Achievement and motivation in boys and girls. Field studies and methodological approaches. Springer.

Organisation for Economic Co-operation and Development, OECD. (2016). PISA 2015 Results (Volume I): Excellence and Equity in Education. OECD Publishing. https://doi. org/10.1787/9789264266490-en

Organización para la Cooperación y el Desarrollo Económicos, OCDE. (2014). Resultados PISA 2012 en foco: Lo que los alumnos saben a los 15 años de edad y lo que pueden hacer con lo que saben. OCDE. https://www.oecd.org/pisa/keyfindings/ PISA2012_Overview_ESP-FINAL.pdf

Organización de las Naciones Unidas para la Educación, la Ciencia y la Cultura, Unesco. (2017). Educación para los Objetivos 
de Desarrollo Sostenible. Objetivos de aprendizaje. Unesco. https://web.unican.es/unidades/igualdad/SiteAssets/ guia-de-recursos/responsabilidad-social-universitaria/ EdS_ODS.pdf

Organización de las Naciones Unidas para la Educación, la Ciencia y la Cultura, Unesco. (2013). Situación educativa de América Latina y el Caribe: Hacia la educación de calidad para todos al 2015. OREALC/Unesco Santiago. http://www.unesco.org/ new/fileadmin/MULTIMEDIA/FIELD/Santiago/images/ SITIED-espanol.pdf

Petit, M. (2003). Nuevos acercamientos a los jóvenes y la lectura. Fondo de Cultura Económica.

Petscher, Y. (2010). A meta-analysis of the relationship between student attitudes towards reading and achievement in reading. Journal of Research in Reading, 33(4), 335-355. https://doi. org/10.1111/j.1467-9817.2009.01418.x

Programa de las Naciones Unidas para el Desarrollo, PNUD. (2017). DESIGUALES. Orígenes, cambios y desafíos de la brecha social en Chile. Uqbar Editores.

Rockwell, E. (2001). La lectura como práctica cultural: conceptos para el estudio de los libros escolares. Educação e Pesquisa, 27(1), 11-26. https://doi.org/10.1590/s1517-97022001000100002

Rodríguez, B., Mahias, P., Maira, M., González, M., Cabezas, H., y Portigliati, C. (2016). La mirada de los profesores: debilidades que reconocen en su práctica y cómo proponen superarlas. MIDE-UC. https://www.mideuc.cl/wp-content/ uploads/2016/04/IT1601-La-mirada-de-los-profesoresAEP-2014.pdf

Rosenblatt, L. M. (1985). Viewpoints: Transaction versus interaction: A terminological rescue operation. Research in the Teaching of English, 19(1), 96-107. https://www.jstor.org/stable/40171006

Sánchez, E., García, R., y Rosales, J. (2010). La lectura en el aula: Qué se hace, qué se debe hacer y qué se puede hacer. Graó.

Shanahan, T. y Shanahan, C. (2012). What is disciplinary literacy and why does it matter? Topics in Language Disorders, 32(1), 7-18. https://doi.org/10.1097/tld.0b013e318244557a

Schraw, G. y Bruning, R. (1999). How implicit models of reading affect motivation to read and Reading engagement. Scientific 
Studies of Reading, 3(3), 281-302. https://doi.org/10.1207/ s1532799xssr0303_5

Street, J. C. y Street, B. V. (1995). The schooling of literacy. En P. Murphy, M. Selinger, J. Bourne, y M. Briggs (Eds.), Subject learning in the primary curriculum: Issues in English, Science and Mathematics (pp. 72-86). Routledge.

Stutz, F., Schaffner, E., y Schiefele, U. (2016). Relations among reading motivation, reading amount, and reading comprehension in the early elementary grades. Learning and Individual Differences, (45), 101-113. https://doi.org/10.1016/j.lindif.2015.11.022

Sulentic-Dowell, M., Beal, G., y Capraro, R. (2006). How do literacy experiences affect the teaching propensities of elementary pre-service teachers? Reading Psychology, 27(2-3), 235-255. https://doi.org/10.1080/02702710600642509

Tolchinsky, L. y Simó, R. (2001). Escribir y leer a través del currículum. ICE-Horsori.

Valenzuela, J. P., Bellei, C., y Ríos, D. (2014). Socioeconomic school segregation in a market-oriented educational system. The case of Chile. Journal of Education Policy, 29(2), 217-241. https:// doi.org/10.1080/02680939.2013.806995

Valenzuela, J. P., Vera, G., y Sotomayor, C. (2015). The role of reading engagement in improving national achievement: An analysis of Chile's 2000-009 PISA results. International Journal of Educational Development, (40), 28-39. https://doi.org/10.1016/j. ijedudev.2014.11.011

Wells, G. y Barberán, S. (2001). Indagación dialógica hacia una teoría y una práctica socioculturales de la educación. Paidós.

Wenger, E. (1998). Communities of practice: Learning, meaning, and identity. Cambridge University Press.

Winocur, R. (2015). Prácticas tradicionales y emergentes de lectoescritura en jóvenes universitarios. En N. García Canclini, V. Gerber, A. López, E. Nivón, C. Pérez, C. Pinochet, y R. Winocur (Eds.), Hacia una antropología de los lectores (pp. 243-281). Ediciones Culturales Paidós.

Yubero, S., Larrañaga, E., y Cerrillo, P. C. (2004). El valor de la lectura: un análisis de la imagen social del lector. En E. Martos y T. Rösing (Eds.), Prácticas de lectura y escritura (pp. 115-136). Editora Universitaria. 
Zavala, V. (2011). La escritura académica y la agencia de los sujetos. Cuadernos Comillas, (1), 52-66. 